\title{
La poétique narrative et descriptive de Jean-Philippe Toussaint : le réel comme oubli de soi
}

\author{
Nicolas Xanthos \\ Université du Québec à Chicoutimi
}

Référentielle, intertextuelle, phénoménologique, la question du rapport au réel dans la fiction se pose de multiples façons. À hauteur de genre, le rapport au réel se complique de toutes les limites et possibilités du signe, le statut de la fiction balançant entre un autotélisme régulièrement revendiqué et une nécessité de dire le monde non moins régulièrement affirmée. À ce chapitre, le propre du contemporain est sans doute de chercher non plus tant à opter pour l'un ou l'autre qu'à intégrer 
un savoir et une mémoire des formes et à en faire, précisément, le moyen d'une transitivité repensée.

À hauteur de diégèse, le réel devient ce que narrateurs et personnages expérimentent, éprouvent, ce à quoi ils se confrontent, ce qu'ils perçoivent - ou ne parviennent pas à percevoir, dans certains cas - , ce à quoi ils donnent sens et qui va, en retour, les définir. Ce sont les modalités de construction du sujet et de l'agent qui s'expriment dans cette expérience fictive du réel, qui se donne à lire à même l'organisation discursive, descriptive et narrative des fictions.

Si l'on a beaucoup, et à juste titre, insisté sur la dimension formelle ou ludique des romans de Jean-Philippe Toussaint ${ }^{1}$, on a un peu moins envisagé son œuvre comme posant la question diégétique du rapport au réel. Or, l'un des principaux intérêts de l'œuvre romanesque de Jean-Philippe Toussaint réside selon nous dans la mise en scène d'une expérience du réel tout à fait particulière, que nous aimerions chercher à définir dans les lignes qui viennent, en une exploration de la poétique romanesque attentive autant aux modalités du récit qu'à celles de la description et à leurs implications philosophiques.

Pour commencer à se faire une idée de cette expérience du réel, on peut procéder à la comparaison de deux courts fragments. On se souviendra des presque dernières lignes du Père Goriot, lorsque Rastignac contemple seul Paris du haut du cimetière du Père-Lachaise :

Rastignac, resté seul, fit quelques pas vers le haut du cimetière et vit Paris tortueusement couché le long des deux rives de la

1 Cette «dimension ludique, faire de virtuosité allusive et de raffinements "méta-" et "intertextuels" » dont parlent, notamment, Dominique Viart et Bruno Vercier (2005, p. 17). 
Seine, où commençaient à briller les lumières. Ses yeux s'attachèrent presque avidement entre la colonne de la place Vendôme et le dôme des Invalides, là où vivait ce beau monde dans lequel il avait voulu pénétrer. Il lança sur cette ruche bourdonnante un regard qui semblait par avance en pomper le miel, et dit ces mots grandioses : "À nous deux maintenant!" (p. 434-435)

Dans les presque dernières lignes de L'Appareil photo de Toussaint ${ }^{2}$, on trouve le narrateur assis dans une cabine téléphonique perdue au milieu de la campagne, où il a abouti un peu par hasard, à pied :

Le jour se levait maintenant, je le voyais se lever derrière les parois de la cabine, c'était encore la nuit, mais une nuit déjà atténuée d'aube claire et bleutée, rien ne bougeait dans la campagne avoisinante, et le jour se levait lentement sous mes yeux, enrobant peu à peu l'air alentour de teintes lumineuses et légères qui enveloppaient l'atmosphère de clarté transparente et tremblante, et, assis derrière les vitres de cette cabine téléphonique complètement isolée dans la campagne déserte, je regardais le jour se lever et songeais simplement au présent, à l'instant présent, tâchant de fixer encore une fois sa fugitive grâce [...] (AP, p. 126-127)

On a visiblement affaire ici à deux expériences du réel radicalement différentes, et à plus d'un titre. La posture physique des deux personnages dit déjà assez les rapports distincts qu'ils entretiennent au réel. Rastignac, lui, est debout et surplombe ledit réel, alors que le narrateur de L'Appareil photo est assis dans une cabine téléphonique. Le premier va s'imposer au réel, alors que le réel s'impose au second. De fait, ils développent l'un et l'autre des rapports à l'action et à l'intention tout à fait dissemblables. Rastignac possède un but

2 Jean-Philippe Toussaint, L'Appareil photo, Paris, Les Éditions de Minuit, 1988. Dorénavant désigné à l'aide du sigle $(A P)$, suivi du numéro de la page. 
clair, sait à quels changements il veut soumettre l'ordre du monde et va tout faire pour les obtenir. Il se situe dans un rapport de lutte avec le réel, l'agir est pour lui une manière de modifier la matière du monde, de la plier à ses desseins. Rien de tel chez le narrateur de L'Appareil photo, qui va bien plutôt tâcher de saisir la fugitive grâce de l'instant présent, autrement dit qui va tenter de laisser s'imposer à lui l'ordre du monde. Le réel n'est pas pour lui le lieu ou le moyen de son affirmation : il ne cherche pas à lui donner sens ou forme selon des intentions définies, mais veut tout autrement saisir au plus près ses agencements intimes et sereins. On peut faire un pas de plus en constatant l'humanisation du réel balzacien auquel Rastignac veut régler son compte. Il faudrait même parler de double humanisation, ici. En effet, de manière évidente, le lieu regardé par Rastignac est d'abord un lieu pensé, construit, organisé, habité par l'être humain : il s'agit de Paris. La spatialité de ce lieu est même déjà structurée en fonction du désir du personnage, qui parvient à définir une aire signifiante. Mais il faut plus encore souligner la consubstantialité du réel et du personnage, l'un et l'autre saisis dans et par la même métaphore apicultrice : réel et personnage sont du coup de la même matière, ils ne sont pas distincts l'un de l'autre, séparés, hétérogènes. Ils sont un seul et même lieu de sens. Le réel est déjà de l'ordre humain, déjà de l'ordre du sens. C'est peut-être pour cela que l'intention du personnage peut s'exprimer aussi aisément: il veut certes changer quelque chose de l'ordre du monde, mais, dans son fondement, cet ordre n'est pas radicalement autre - et l'intention humaine s'y trouve en terrain connu. Le réel de Toussaint, en revanche, n'est pas humain - comme le marque la coupure instaurée par la vitre de la cabine téléphonique - sans pour autant lui être hostile, 
loin de là. Il est d'abord une couleur, une teinte, ou mieux encore : une qualité de la lumière qui se déploie dans toute sa fragilité, petit miracle qui aurait pu passer inaperçu, spectacle indifférent à ses spectateurs. Il se déploie dans les marges du monde humain du sens et exige une attention toute particulière pour pouvoir être appréhendé, une attention que nous verrons plus tard comme un oubli de soi. La temporalité des expériences est aussi totalement différente. Conformément à ce qu'implique l'idée d'intention, Rastignac pense son rapport au réel dans la durée, de manière prospective : le réel, c'est ce qu'il va transformer. Lorsqu'il dit : «À nous deux maintenant! », c'est à partir de maintenant, et pour longtemps — tant et aussi longtemps qu'à l'ordre des choses ne se sera pas imposé l'ordre du personnage. On pourrait d'ailleurs ajouter que ce rapport au réel prospectif est aussi le fruit d'un rapport au réel rétrospectif : c'est d'avoir échoué qu'il s'apprête à reprendre sa tâche. Bref, c'est maintenant le temps qui se trouve structuré par le désir du personnage, tout comme l'était précédemment l'espace. Rien de tel chez Toussaint: le narrateur se concentre sur le seul instant présent, épuré, débarrassé tout à la fois de ses héritages et de ses prolongements, et qui vit à la périphérie des desseins humains.

Ce sont en définitive deux univers fictionnels totalement distincts que nous venons de voir quant à la question de l'expérience du réel. Pour synthétiser de manière un peu cavalière, on pourrait dire que, chez Balzac, le réel est le lieu humanisé de l'action, alors que, chez Toussaint, il est cette altérité apaisée qui se perçoit sitôt que l'on parvient à renoncer à soi comme manière d'imposer du sens. L'hypothèse qui va nous guider maintenant, c'est que la poétique de Toussaint est tout entière informée par ce désir, ou ce vœu, de renoncement à 
soi pour, précisément, parvenir à faire l'expérience du réel. On verra également que cette expérience a pour but de s'opposer à la douloureuse corruption qu'impose le passage du temps.

Posons déjà qu'on retrouve cette expérience du réel dans tous les romans de Toussaint: elle s'en fait la forme la plus aboutie, ce vers quoi tend la construction romanesque dans son ensemble, dont elle conditionne en profondeur la poétique.

Qu'on pense à Monsieur ${ }^{3}$, lorsque le personnage principal observe le ciel :

Autour de Monsieur, maintenant, c'était comme la nuit même. Immobile sur sa chaise, la tête renversée en arrière, il mêla de nouveau son regard à l'étendue des cieux, l'esprit tendu vers la courbure des horizons. Respirant paisiblement, il parcourait toute la nuit de la pensée, toute, loin dans la mémoire de l'univers, jusqu'au rayonnement du fond du ciel. Atteignant là l'ataraxie, nulle pensée ne se meut plus alors dans l'esprit de Monsieur, mais son esprit était le monde - qu'il avait convoqué.

Oui. Il allait se gêner, Monsieur. (M, 96-97)4

Dans La Salle de bain ${ }^{5}$, le narrateur est, à Venise, au restaurant devant ce dessert glacé qu'est la dame blanche, et il contemple le tout :

Je regardais la dame blanche fondre devant moi. Je regardais fondre imperceptiblement la vanille sous la nappe de chocolat brûlant. Je regardais la boule encore exactement ronde un instant plus tôt qui ruisselait lentement en filets blancs et bruns métissés. Je regardais le mouvement, immobile, les yeux fixés

3 Id., Monsieur, Paris, Les Éditions de Minuit, 1992. Dorénavant désigné à l'aide du sigle $(M)$, suivi du numéro de la page.

4 Signalons que ce passage a été finement commenté par François Ouellet (1996).

5 J.-Ph. Toussaint, La Salle de bain, Paris, Les Éditions de Minuit, 1985. Dorénavant désigné à l'aide du sigle $(S B)$, suivi du numéro de la page. 
sur la soucoupe. Je ne bougeais pas. Les mains figées sur la table, j'essayais de toutes mes forces de garder l'immobilité, de la retenir, mais je sentais bien que, sur mon corps aussi, le mouvement s'écoulait. (SB, p. 80)

Et il est impossible de ne pas mentionner la baignade nocturne du narrateur de Faire l'amour ${ }^{6}$ sur le toit de son hôtel, à Tokyo.

Je nageais comme en apesanteur dans le ciel, respirant doucement en laissant mes pensées se fondre dans l'harmonie de l'univers. J'avais fini par me déprendre de moi, mes pensées procédaient de l'eau qui m'entourait, elles en étaient l'émanation, elles en avaient l'évidence et la fluidité, elles s'écoulaient au gré du temps qui passe et coulaient sans objet dans l'ivresse de leur propre écoulement, la grandeur de leur cours, comme des pulsations sanguines inconscientes, rythmées, douces et régulières, et je pensais, mais c'est déjà trop dire, non, je ne pensais pas, je faisais maintenant corps avec l'infini des pensées, j'étais moi-même le mouvement de la pensée, j'étais le cours du temps. (FA, p. 51-52).

Ces scènes manifestent une manière d'être, d'agir et de percevoir le réel où résident précisément les modalités de la poétique narrative et descriptive de Toussaint.

\section{Poétique narrative}

Dans Faire l'amour, le narrateur, aux prises avec un méchant rhume, passe à un moment donné quelques jours à Kyoto chez un ami. Le voilà qui se lève, un matin.

Je venais d'émerger d'un sommeil de près de trente-six heures, entrecoupé de brèves allées et venues de mon lit à la cuisine, et, croyant être toujours seul dans la maison, j'étais sorti de la chambre pour aller prendre le petit déjeuner en me rajustant

${ }^{6}$ Id., Faire l'amour, Paris, Les Éditions de Minuit, 2002. Dorénavant désigné à l'aide du sigle $(F A)$, suivi du numéro de la page. 
nonchalamment les couilles dans mon caleçon fané (quel homme d'action, vraiment). . (FA, p. 152-153)

La remarque ironique finale peut s'appliquer à tous les narrateurs ou personnages principaux des romans de Toussaint. Ils entretiennent systématiquement un rapport pour le moins lâche et flou à l'action et à l'intention. Nous n'avons pas là des fonceurs, d'habiles planificateurs, des décideurs, des êtres engagés politiquement ou socialement, des personnages déterminés, etc. Ils n'ont pas véritablement d'intention qui structure leur trajectoire romanesque, qui donne sens à leurs menus gestes en en faisant les parties d'un tout où ils pourraient recevoir place et sens : ils subissent l'action d'autrui (ou n'agissent pas), mais, d'eux-mêmes, ils font bien peu de choses. Ils sont souvent entraînés ou poussés par quelqu'un d'autre, qui les entraîne dans certaines péripéties: c'est Edmondsson pour le narrateur de La Salle de bain; Marie pour celui de Faire l'amour; Il signore Gambini ou Monsieur Polougaïevski pour celui de L'Appareil photo; le scientifique Kaltz pour Monsieur; Zhang Xiangzhi et Li Qi pour le narrateur de Fuir ${ }^{7}$. De façon spectaculaire (les frasques à moto dans Pékin) ou dérisoire (la recherche d'une bouteille de gaz au supermarché, dans une voiture qui tombe en panne), ils se trouvent insérés dans les plans et actions d'autrui, y consentent de plus ou moins bon gré sans se sentir nécessairement toujours concernés ou impliqués par ce qui se passe. Il leur arrive d'ailleurs souvent d'abandonner l'action en cours ou de s'en faire expulser. De leur propre chef, ils ne font pas grandchose, n'ont pas de but défini, ne se projettent pas dans l'avenir. Très souvent, ils ne font rien, restent immobiles, au lit, dans une

7 Id., Fuir, Paris, Les Éditions de Minuit, 2005. Dorénavant désigné à l'aide du sigle $(F)$, suivi du numéro de la page. 
baignoire, sur le bord de la plage, ou alors, ils se déplacent mais au hasard (la mention est fréquente dans les textes), sans trop savoir où aller. De façon tout à fait symptomatique, le narrateur le plus actif des romans de Toussaint est sans doute celui de $L a$ Réticence ${ }^{8}$, arrivé au village de Sasuelo à la fin octobre pour aller voir des amis, les Biaggi, mais qui ne se résout pas à prendre concrètement contact avec eux.

C'était en quelque sorte pour voir les Biaggi que je m'étais rendu à Sasuelo, mais, jusqu'à présent, retenu par une espèce d'appréhension mystérieuse, j'avais toujours retardé le moment de leur rendre visite, évitant même les parages de leur maison quand je me promenais dans le village. Le jour de mon arrivée déjà, alors que je pensais passer chez eux sitôt installé à l'hôtel, j'avais sans cesse différé le moment d'aller les trouver, et j'étais resté tout l'après-midi dans ma chambre. (R, p. 16)

On ne manquera pas non plus de mentionner les ambitieux projets d'écriture du narrateur de La Télévision ${ }^{9}$. Ce professeur en congé sabbatique à Berlin grâce à une généreuse subvention souhaite en effet rédiger un essai sur la rencontre $\mathrm{du}$ Titien et de Charles-Quint et ses implications politicoartistiques. L'écriture de l'ouvrage ne progresse pas et l'une des seules références immédiates qu'on y trouve tourne autour d'une phrase que le narrateur commence à écrire ("Quand Musset, abordant dans sa nouvelle... » [T, p. 84]), puis dont il ne conserve que ce qui lui semble parfait ("Quand Musset»). Fort aise de cette première pierre sur laquelle bâtir sa cathédrale, il la prononce à haute voix: "Je le dis un peu plus fort. Quand Musset. Je m'accoudai à la balustrade du balcon et le gueulai un

\footnotetext{
${ }^{8}$ Id., La Réticence, Paris, Les Éditions de Minuit, 1991. Dorénavant désigné à l'aide du sigle $(R)$, suivi du numéro de la page.

9 Id., La Télévision, Paris, Les Éditions de Minuit, 2002 [1997]. Dorénavant désigné à l'aide du sigle $(T)$, suivi du numéro de la page.
} 
petit coup : Quand Musset! Quand Musset! répétais-je au balcon. Quand Musset! Silence! entendis-je soudain, silence, s'il vous plaît! Cela venait d'en bas. » ( $T$, p. 85) Par-delà le comique irrésistible de l'épisode (comme du roman), il faut bien voir le processus de réduction (au silence) qui est à l'œuvre ici sur le plan de l'action : le projet devient une simple circonstancielle sans même un verbe conjugué, totalement périphérique et incapable d'en venir aux faits, puis se limite à deux mots au rapport encore plus lâche avec lui et, en eux-mêmes, assez peu susceptibles d'entraîner la satisfaction, pour se faire finalement sanctionner d'un rappel à l'ordre qui agit comme une sanction de toute la péripétie et impose le silence.

À sa manière, L'Appareil photo se fait la fable de cette défaillance de l'agir. Dans les premières pages, le narrateur explique le sens de son jeu d'approche envers une jeune femme, dans un propos de portée générale :

[...] tout mon jeu d'approche, assez obscur en apparence, avait en quelque sorte pour effet de fatiguer la réalité à laquelle je me heurtais $[\ldots]$, et $[\ldots]$ ma propension à ne jamais rien brusquer, bien loin de m'être néfaste, me préparait en vérité un terrain favorable où, quand les choses me paraîtraient mûres, je pourrais cartonner. (AP, p. 14)

Ce commentaire «métaactionnel» explique la façon d'être agent du narrateur du roman. Il ne refuse pas l'action, ne s'en tient pas éloigné, tout différemment: ce qu'il fait, ou plus exactement ne fait pas, a pour fonction de rendre les circonstances propices à une action encore à venir. Il ne se situe donc pas hors de la sphère actionnelle, même s'il joue le jeu de façon un peu inattendue. Plus encore, il possède un but clair qui oriente son (in)action. Et il semble d'abord que cette stratégie 
inhabituelle porte ses fruits, comme le narrateur l'évalue plus tard :

[...] je sentais confusément que la réalité à laquelle je me heurtais commençait peu à peu à manifester quelques signes de lassitude; elle commençait à fatiguer et à mollir oui, et je ne doutais pas que mes assauts répétés, dans leur tranquille ténacité, finiraient peu à peu par épuiser la réalité [...] et que lorsque, exténuée, la réalité n'offrirait enfin plus de résistance, je savais que plus rien ne pourrait alors arrêter mon élan, l'élan furieux que je savais en moi depuis toujours, fort de tous les accomplissements. (AP, p. 50)

On paraît bien loin ici des postures de patient, de retrait dans la pensée ou de disparition : c'est même tout le contraire, puisque le narrateur envisage le surgissement, dans le monde, de son élan furieux et impossible à arrêter. C'est une pleine présence dans le monde par le biais de l'action qui est ici projetée, un monde auquel l'intention de l'agent pourra imposer son ordre. C'est, à son meilleur quoique encore en puissance, l'homme d'action, stratège et volontaire, qui pointe le bout de son nez.

Cela ne dure pas, toutefois. À peine quelques pages plus loin, le narrateur fait ce nouveau constat :

[...] la réalité à laquelle je me heurtais, bien loin de marquer le moindre signe d'essoufflement, semblait s'être peu à peu durcie autour de moi et, me trouvant désormais dans l'incapacité de m'extraire de cette réalité de pierre qui m'entourait de toutes parts, je voyais à présent mon élan comme un surgissement de forces arrachantes à jamais prisonnier de la pierre. (AP, 56)

Il n'est plus question maintenant d'imposer son ordre à la réalité : c'est elle qui, bien plutôt, impose son ordre au narrateur, l'immobilisant et figeant son élan à même sa nature minérale - le durcissement de la pierre ayant remplacé, dans le registre métaphorique, la molle fatigue de tout à l'heure. 
Quant au narrateur, sa position active d'assaillant s'est transformée en mauvaise posture d'assiégé.

On s'en souviendra, lorsque le narrateur traverse la Manche pour retourner en France, il trouve dans la cantine du ferry un petit appareil photo qu'il prend, puis se trouve à voler malgré lui. Dans la précipitation du geste, il court sur le bateau, prend une volée de photos, s'empare de la pellicule et jette l'appareil à la mer. Plus tard, en rêvant à un autoportrait qui rendrait compte de sa seule présence, il se rend compte qu'il l'a fait, cette nuit-là, lors de la traversée :

[...] c'est sur le bateau que j'avais fait cette photo, [...] presque inconscient d'être en train de photographier et pourtant me délivrant de cette photo à laquelle j'aspirais depuis si longtemps et dont je comprenais à présent que je l'avais saisie dans la fulgurance de la vie, alors qu'elle était inextricablement enfouie dans les profondeurs inaccessibles de mon être. C'était comme la photo de l'élan furieux que je portais en moi [...]. (AP, p. 113)

La capacité d'agir semble ne pas être totalement annihilée. Quand bien même le contexte donne peu l'impression qu'il s'agit d'une action réfléchie, il n'en reste pas moins que ces photos prises sur le bateau paraissent porter en elles l'attestation d'une force intentionnelle capable de s'imposer dans le monde et d'y imposer quelque chose de l'être. La disparition n'est dès lors plus la seule manière d'exister. C'est donc plein de cet espoir que le narrateur va faire développer cette pellicule. Hélas, le verdict est sans appel: aucune des photos prises cette nuit-là n'a pu être développée, la pellicule étant sous-exposée. Seules demeurent sur les négatifs « çà et là quelques ombres informes comme d'imperceptibles traces de mon absence » (AP, p. 116). Devant cet échec de l'agir consacré 
jusque dans ses manifestations les plus minimales, on ne s'étonnera pas de retrouver, à la toute fin du texte, le passage sur lequel nous avons ouvert notre réflexion : le narrateur assis dans une cabine téléphonique au milieu de la campagne, passant la nuit perdu dans ses pensées « les yeux fermés et le corps à l'abri » (AP, p. 125), puis « regard[ant] le jour se lever et songe[ant] simplement au présent, à l'instant présent, tâchant de fixer encore une fois sa fugitive grâce » $(A P$, p. 127), pur patient, en somme.

Le portrait intentionnel de ces personnages laisse donc voir des êtres qui n'ont pas d'ordre particulier à imposer au monde, qui ne cherchent pas à modifier l'état des choses, qui ne veulent pas imprimer leur marque dans le réel par l'agir. Ils se situent tous dans l'orbe de cette ataraxie évoquée dans Monsieur. L'être, visiblement, ne se définit pas par son action, son intention. À côté, donc, du j'agis donc je suis, ou du je parle donc je suis, s'élabore une anthropologie marginale où l'on n'est ni homme d'action ni homme de parole. Restera à voir quel homme s'énonce dans ce refus de l'agir.

Sur le plan de l'organisation narrative, cette absence d'intention se traduit par une intrigue faiblement configurée, où la discordance des péripéties n'est pas subsumée par la concordance de l'entier de la configuration narrative ${ }^{10}$. Les épisodes dont se constituent les romans se suivent souvent sans nécessité affirmée ${ }^{11}$. À ce titre, le principe de structuration

\footnotetext{
10 Voir à ce propos, chez Ricœur le chapitre consacré aux métamorphoses de l'intrigue (1984, p. 17-48).

11 Sur cette question et pour constater ses liens avec la question intentionnelle, on pourra lire, en parallèle avec nos précédents commentaires, les commentaires que Marie-Pascale Huglo (2007, p. 96-100) consacre à la structure narrative de L'Appareil photo.
} 
affiché par La Salle de bain continue d'opérer souterrainement dans les autres romans. On se souviendra que, dans ce premier texte, le récit est découpé en paragraphes ou ensembles de paragraphes numérotés, ce qui donne l'impression d'une simple succession, sans que les segments ne soient insérés dans un ordre plus vaste, faisant de chacun d'eux la partie d'un tout qui, en retour, les rendrait signifiants. D'une certaine manière, on pourrait se dire ici proche de la chronique - à quoi, on s'en souvient, Aristote opposait la mise en intrigue propre à l'art du poète ${ }^{12}$. Le principe de la chronique est qu'on suit non pas une action, de son début logique jusqu'à sa fin logique, mais une période de temps seulement, plus ou moins arbitrairement déterminée, qui doit son unité à la seule surface temporelle qu'elle couvre. Il y a du reste là une correspondance entre l'activité énonciative du narrateur et la manière d'être du personnage (narrateurs et personnages qui, le plus souvent, coïncident) : tout comme le personnage ne pense pas son rapport au réel sur le mode de l'action, le narrateur ne pense pas sa relation d'événements sur le mode de la mise en intrigue. À l'intention nébuleuse du personnage correspondent le récit faiblement configuré du narrateur, son attention soutenue témoignée à des éléments apparemment secondaires, ses choix narratifs qui semblent manquer ce qu'on aimerait dire ailleurs essentiel, comme dans cet exemple tiré de La Salle de bain, où le narrateur, dans la baignoire, accueille un ami de ses parents :

\footnotetext{
12 Aristote oppose sous deux aspects l'art du poète et celui de l'historien. Un premier dans le chapitre IX de la Poétique, qui nous intéresse peu ici, est celui du vraisemblable et du vrai. Un second dans le chapitre XXIII, autour de l'unité de temps qui fonde la pratique de l'historien (et penche du côté de la chronique) et de l'unité d'action qui fonde celle du poète (avec une forte configuration narrative). On lira sur cette question l'intéressante réflexion de Bérenger Boulay (2008).
} 
[...] il me donna des nouvelles de ses activités professionnelles, m'expliquant que les difficultés auxquelles il se heurtait étaient insurmontables, car liées à des incompatibilités d'humeur entre des personnes de même niveau hiérarchique. Jouant nerveusement de ma serviette, il marchait à grands pas le long de la baignoire et, exalté par ses propos, se montrait de plus en plus intransigeant. Il menaçait, vociférait. Finalement, il traita Lacour d'irresponsable. Je tente l'impossible, disait-il, l'impossible! et tout le monde s'en fout. (SB, p. 14)

Quelles difficultés, qui est Lacour, qui menaçait-il, que tentait-il? De tout cela, qui pourtant semble non dénué d'intérêt dans l'anecdote, le lecteur ne saura rien, en raison de l'emploi du discours narrativisé et de la discrétion du discours direct. En utilisant une métaphore empruntée à la photographie ou au cinéma, on pourrait dire que le cadrage narratif est étrangement construit: on met l'accessoire au centre de la scène et l'essentiel hors champ - ou du moins ce qui aurait pu paraître essentiel et accessoire à l'intérieur d'une narration où primeraient la représentation de l'action et l'intention fortes, qui permettraient de valoriser les éléments de la scène et de structurer cette dernière en fonction des impératifs de progression de l'intrigue. C'est un refus de l'intrigue conventionnelle que manifestent ces fictions, sinon un refus du romanesque, tout à la fois comme forme cohérente et comme contenu pompier.

Bref, cette ouverture à l'expérience du réel se traduit, sur le plan narratif, par des personnages aux intentions pour le moins nébuleuses - pour ne pas dire dépourvus d'intentions - entraînés malgré eux dans les actions d'autrui, et par des intrigues si faiblement configurées qu'elles confinent à l'abandon d'un certain romanesque. C'est, de la sorte, en refusant d'imposer au monde ou à la matière fictionnelle un 
ordre né de l'intention ou de la configuration narrative, un sens fort, que Toussaint crée les conditions favorables à une mise en scène de l'expérience du réel.

\section{Poétiques descriptives}

Bien que la pratique descriptive de Toussaint semble répondre à plusieurs impératifs et se présente du coup sous des dehors plus nuancés qui résistent à l'unification, on peut y lire des enjeux similaires à ceux qu'on a observés à l'instant et qui touchent à l'oubli de soi. Ils y prennent deux formes, selon que le regard porté sur le monde permette cet oubli ou qu'au contraire, s'impose à lui, dans la matière du monde, la douleur $\mathrm{du}$ passage du temps. Le premier type de description, géométrique et lumineux, abstrait, spatial, possède souvent des dehors apaisés ou apaisants, alors que le second type de description, marqué par le concret du débris, manifeste une mélancolie désespérée. La coprésence de ces deux regards sur le réel montre bien la nécessité de la disparition identitaire dont on suit la trace : c'est pour parer aux blessures du temps que le narrateur se perd dans une géométrie lumineuse ${ }^{13}$. Observons tour à tour ces deux poétiques descriptives.

Pour le redire en peu de mots, les aspects ou dimensions des objets décrits relèvent préférentiellement, dans la première poétique, de ces trois domaines: géométrie des formes, luminosités et mouvements ou immobilités. En voici quelques exemples caractéristiques.

13 « [C]ar la géométrie est indolore, sans chair et sans idée de mort » ( $F$, p. 100). 


\section{Dans La Salle de bain, tout d'abord :}

Sous les rideaux entrouverts, tout au long du mur, la lumière découpait des surfaces, traçait des contours brûlants sur le parquet. Malgré quelques taches de clarté, très vives par endroits, la pièce, tout immobile, restait baignée dans un marais d'obscurité. (SB, p. 72)

\section{Dans Monsieur :}

Souvent, avant de remonter dans son bureau, Monsieur [...] passait quelques instants debout devant l'aquarium et regardait les poissons les mains dans les poches, ne se lassant pas de contempler l'inaccessible pureté des trajectoires qu'ils traçaient avec indifférence. (M, p. 89-90)

\section{Dans La Réticence :}

Le phare de l'île de Sasuelo tournait avec régularité dans la nuit, et tout était parfaitement silencieux autour de moi. J'étais assis là tout seul en manteau sombre sur la plage, les pieds nus dans le sable mouillé, et je vis un bateau apparaître à l'horizon alors, un ferry qui glissait lentement devant moi tout illuminé dans la nuit, qui glissait immobile à la surface de l'eau et qui finit par disparaître très lentement derrière l'île de Sasuelo. (R, p. 159)

\section{Deux exemples, pour finir, dans Faire l'amour :}

De l'endroit du restaurant où nous nous trouvions, on ne voyait dans l'encadrement de bois de la fenêtre qu'un fragment de rue partiel et incohérent qui donnait sur un immeuble dans la pénombre, avec des fils électriques mystérieux et une colonne de lumière qui montait à la verticale le long de la façade, composée de sept ou huit caissons lumineux superposés qui annonçaient la présence de bars à chaque étage du bâtiment. (FA, p. 70)

Nous avancions ainsi à l'inconnu dans de sombres ruelles quand nous aperçûmes sur le trottoir d'en face la cage de verre illuminée d'un petit supermarché ouvert vingt-quatre heures sur vingt-quatre, à l'enseigne bleue et blanche de Lawson qui brillait dans la nuit. Nous allâmes nous abriter un instant à l'intérieur, passant sans transition de la pénombre bleutée de la 
nuit à la violente clarté intemporelle d'un plafonnier de néons blancs. (FA, p. 74)

Quel que soit l'objet qui fait les frais de la description (qu'il s'agisse d'un objet naturel ou de la main de l'homme), ses modalités de saisie relèvent, en proportions variables mais presque systématiquement, des formes, des luminosités et des mouvements. En un sens, on pourrait dire qu'il s'agit d'une saisie minimale, qui ne cherche pas à intégrer l'objet dans un univers de sens - qu'il s'agisse d'un sens social ou d'un sens individuel. L'objet est ainsi saisi dans une sorte d'instantanéité qui le coupe de toute réverbération dans la biographie du descripteur, dans le passé social ou culturel du monde qui l'entoure ou $\mathrm{du}$ monde d'où il vient. Aucune destinée individuelle ou collective n'est projetée sur lui, ne vient l'informer, ne vient s'y refléter pour mieux s'y exprimer. Chez Toussaint, l'objet n'est pas une terre d'accueil pour l'histoire des hommes.

L'objet de la description est presque systématiquement perçu par la vue, qui, on s'en souviendra, est des cinq sens celui de la plus grande distance. Et s'il rencontre quelque chose du descripteur humain, c'est par ce sens-là : la luminosité, si souvent mise de l'avant, est avant tout une sensation. De la conscience humaine, la portion sensible (liée à la sensation) est la seule à être convoquée lors de ces opérations descriptives : aucun savoir, aucun jugement, aucune affectivité ne sont exploités ici, qui pourraient aller au-delà de l'objet, l'intégrer dans des univers de sens intimes ou collectifs, qui pourraient, pour ainsi dire, nous voir en lui. Autant on pouvait voir Rastignac dans Paris, autant il est impossible de se retrouver dans les objets de Toussaint. Ne pouvant nous y retrouver, nous ne pouvons que nous y perdre: c'est effectivement une 
contemplation où s'abîme le sens commun que mettent en scène les descriptions de Toussaint. Le monde est laissé à luimême, il ne possède plus, chez Toussaint, la forme de nos destins ni nos souffrances. Sa géométrie, ses immobilités ou ses mouvements, ses luminosités sont l'oubli de l'humain. Ou, pour le dire autrement, le percevoir et le décrire comme forme, mouvement et luminosité, c'est un oubli de soi : c'est chercher à effacer ce lien de sens que l'acte descriptif a si souvent tenté d'instaurer entre l'homme et le monde et qui faisait que nos histoires ou nos savoirs donnaient sens et forme au monde ${ }^{14}$.

Le discours de savoir sur le monde est du reste parodié dans Monsieur, puisque le personnage principal devient, évidemment malgré lui, le secrétaire d'un géologue qui lui dicte des descriptions de minéraux, souvent reproduites dans le roman. S'il s'agit bien d'un discours scientifique dépourvu d'affectivité et si la géométrie des formes est souvent mise à contribution, il n'en reste pas moins que l'opération d'imposition de sens est constante, tant sur le plan lexical que sur le plan discursif. On ne cherche pas là à s'ouvrir à une expérience du réel : on saisit des morceaux caillouteux de réel pour les cataloguer. Et ce n'est pas un hasard si le scientifique en question, Kaltz, apparaît dans le roman sous des dehors peu flatteurs et que, finalement, Monsieur cessera de lui servir de secrétaire : ce rapport de savoir au monde n'est pas celui que privilégie l'univers fictionnel de Toussaint.

Tout à la fois géométrique et sensible, cette première poétique descriptive de Toussaint vient à sa manière dire le rapport au réel de ces fictions : le réel n'y est pas soumis à un

\footnotetext{
14 On pense ici à la pratique de la description expressive et de la description représentative. Voir à ce propos Adam et Petitjean (1989).
} 
ordre signifiant (qu'il soit intime ou social, historique ou scientifique) qui le structure humainement du dehors et se l'annexe; tout différemment, mais sans hostilité, il est à distance, possède ses propres architectures, est indifférent au sens et peut être visuellement éprouvé. On peut s'y exposer (et il faut entendre dans ce verbe un peu de son sens photographique), mais il ne nous expose pas (au sens muséal $\mathrm{du}$ terme). Le décrire ainsi que le fait Toussaint, c'est le regarder pour s'y oublier.

Mais cet oubli ne parvient pas toujours à s'éprouver et, lorsque la manœuvre échoue, c'est le passage du temps qui s'impose au narrateur, en un second type d'expérience descriptive qui prend un tour dysphorique. Dans l'imaginaire des lieux que déploie la fiction de Toussaint, c'est bien souvent la lointaine banlieue qui devient, dans l'espace vécu, le territoire privilégié de cette douloureuse rencontre avec le temps, loin des lumières de la ville. Il en va ainsi de la banlieue parisienne dans L'Appareil photo, ou berlinoise dans La Télévision :

En bordure du lac, aménagé comme un espace de loisir et de détente, les noms des rues et des promenades évoquaient le midi de la France et, parmi cet espace aride et bétonné, était un restaurant désert, avec une terrasse à l'abandon où des parasols refermés prenaient la pluie. Les eaux du lac, comme lasses et paresseuses, venaient se perdre dans la boue d'une manière de plage grisâtre qui se prolongeait en un terrain vague où se trouvait une vieille cabane en bois, branlante et palafitte, avec des planches cloutées abandonnées devant la porte et un wishbone cassé, traînant par terre, dans l'herbe tendre et mouillée de pluie. (AP, p. 66)

$\mathrm{Au}$ bout de cette Rilkestrasse, simple allée en cul-de-sac qui donnait elle aussi dans le vide sidéral de la Gagarine Allee, nous commençâmes à chercher le bloc $\mathrm{D}$, où habitait Ursula, 
l'étudiante de John, en errant au hasard devant les entrées des immeubles, au début ensemble, John et moi, côte à côte, puis séparément, nous aventurant chacun de notre côté parmi ces blocs de béton identiques que différenciaient seulement, çà et là, quelques lettres grisâtres passées qui avaient été peintes au pochoir à côté des entrées, un $\mathrm{E}$ fantomatique, les dernières traces d'un F à demi-effacé. (T, p. 161)

On pourrait ajouter, en ce qui concerne Berlin, l'ancien aéroport nazi, puis soviétique, constitué des traces délabrées de ces époques révolues et presque dépourvu d'existence propre dans le présent.

On le voit, ces descriptions s'attardent essentiellement aux débris qui signalent d'une manière lugubre un passage du temps qui n'est que dégradation et corruption: l'altération déliquescente s'y fait le gris destin des choses (et, derrière elles, des êtres). La vanité des œuvres humaines se répète d'un bout à l'autre de ces espaces qui dépérissent sous les assauts du temps et ne sont que les survivances momentanées et morbides de ce qui a été. Le présent intemporel et immobile des descriptions construites sous le précédent régime le cède maintenant à un présent mélancolique, qui ne vaut que d'accueillir la fatalité de la décrépitude et ne s'ouvre sur aucun avenir, comme à bout de souffle.

À Berlin comme à Sasuelo, du reste, on ne compte plus ces objets abandonnés (le terme, déjà, revenait à deux reprises dans le seul paragraphe de L'Appareil photo cité à l'instant) : "La rangée de boîtes aux lettres avait été arrachée et gisait par terre à la renverse, l'une d'elles, isolée, abandonnée contre un mur, regorgeait de courrier, d'imprimés et de prospectus» $(T$, p. 162); «nous roulions dans un parc désert, abandonné, parmi des fantômes de SS et d'officiers soviétiques » (T, p. 173); «Un 
Mig [...] était abandonné au milieu d'une allée à côté des épaves de quelques camions bâchés, d'un groupe électrogène abandonné dans l'herbe » ( $T$, p. 173); «Tout au long des allées se dressaient d'autres baraquement sordides, d'autres bunkers désaffectés, des blockhaus écroulés [...] où avaient été abandonnés quelques objets métalliques corrodés » ( $T$, p. 173174); «[nous] finîmes par nous arrêter devant un hangar abandonné » $(T, \quad$ p. 174); «C'était une plage immense, abandonnée et déserte » $(R, \mathrm{p} .14)$; « un âne solitaire broutait du fenouil entre divers détritus, des vieilles planches, des pneus abandonnés, une barque retournée qui pourrissait sur place » ( $R$, p. 15); " nous longeâmes au ralenti une église abandonnée, presque en ruines » $(R, \mathrm{p} .32)$; « une fourgonnette blanche [...] était garée [...] devant la façade d'une maison abandonnée » $(R$, p. 47); «quelques chaises cassées étaient entreposées dans la pénombre à côté d'un vieux matelas abandonné par terre » $(R$, p. 71); « des serviettes froissées abandonnées en boule sur les nappes » $(R$, p. 73$)$; « la cage débordait [...] de sacs-poubelles [...], certains ayant été particulièrement choyés avant d'être abandonnés » $(R$, p. 84 ; la décharge publique fera du reste l'objet de plusieurs descriptions dans le roman, toujours dans les mêmes termes); "Il y avait un champ dans l'obscurité en bordure du chemin, un champ abandonné et silencieux que protégeait une vieille clôture tout abîmée [...]» $(R$, p. 158). À tout instant, en tout objet comme en tout lieu, le temps comme déchéance est susceptible d'imposer sa marque et de contraindre le regard descripteur à subir et à enregistrer les innombrables signes de son empire. On comprend mieux, dès lors, le dernier souhait du narrateur de L'Appareil photo de saisir la fugitive grâce de l'instant présent et de l'immobiliser : c'est le souhait d'empêcher l'inéluctable déclin qui, sinon, 
affecte tout et tous. Ce n'est pas plus vers l'avenir que vers le passé que réside, chez Toussaint, l'espoir de l'apaisement : il ne peut se trouver dans que le détachement géométrique d'un présent hors du temps. De là, aussi, ces intrigues, qu'on a dites faiblement configurées et qui sont une manière de préserver les moments du récit, de les autonomiser pour éviter qu'ils n'entrent dans une durée certes signifiante, mais aussi assassine.

Cette expérience douloureuse du temps dans l'espace, contre quoi doit lutter l'oubli de soi, est présente à la toute fin d'Autoportrait (à l'étranger)15. Le narrateur (faut-il dire Toussaint?) est de retour à Kyoto et se retrouve à une station de métro fermée depuis plusieurs semaines :

[...] à la vue de ce spectacle de désolation, de cette gare abandonnée derrière des grilles de fer qui en interdisaient l'accès, cette gare déserte aux quais désaffectés, dont les voies étaient devenues un terrain vague bosselé détrempé par la pluie et la salle des guichets et des appareils de billetterie un dépotoir où reposait encore un tourniquet bancal de guingois dans la boue, je pris conscience que le temps avait passé depuis mon départ de Kyoto. Et, si j'y fus si sensible ce jour-là, [...] c'est aussi parce que je me suis soudain senti triste et impuissant devant ce brusque témoignage du passage du temps. Ce n'était guère le fruit d'un raisonnement conscient, mais l'expérience concrète et douloureuse, physique et fugitive, de me sentir moimême partie prenante du temps et de son cours. (AA, p.118119)

On ne sera pas surpris d'apprendre que cette gare est éloignée du centre de Kyoto, à la périphérie de la ville, tout comme la pratique poétique de Toussaint s'avère une manière de

15 J.-Ph. Toussaint, Autoportrait (à l'étranger), Paris, Les Éditions de Minuit, 2000. Dorénavant désigné à l'aide du sigle $(A A)$, suivi du numéro de la page. 
maintenir, dans l'oubli de soi, la corruption du temps à la périphérie d'une expérience du réel toujours menacée de se transformer, pour un sujet qui désire s'absenter, s'abstraire ${ }^{16}$, se préserver, aussi, en un rappel à l'ordre temporel. Et, quittant la poétique pour la poïétique, les modalités d'un dire romanesque pour ses motivations, ou compliquant celles-là de celles-ci, on citera, pour conclure cette brève excursion dans la description chez Toussaint, les lignes qui suivent immédiatement le dernier fragment et qui terminent le texte :

Jusqu'à présent, cette sensation d'être emporté par le temps avait toujours été atténuée par le fait que j'écrivais, écrire étant en quelque sorte une façon de résister au courant qui m'emportait, une manière de m'inscrire dans le temps, de marquer des repères dans l'immatérialité de son cours, des incisions, des égratignures. (AA, p. 119-120)

\section{L'expérience du réel comme oubli de soi}

Sur les plans narratif et descriptif, les dispositifs de Toussaint semblent viser à une expérience du réel comme oubli de soi, avec le rappel du péril sinon encouru. L'absence d'intention (et le type d'intrigue qui en découle) fait que le personnage ne se positionne pas par rapport au monde, ne l'évalue pas, n'y voit pas des causes d'insatisfaction, n'imagine pas pour ce même réel une forme nouvelle qu'il pourrait lui imposer pour son bénéfice personnel. Il ne veut pas faire le réel à son image, s'y retrouver : il le laisse tel qu'il est, à ses ordres ou désordres aux causes incertaines, mais quoi qu'il en soit indépendant de lui et indifférent à lui. La disparition de l'intention est une disparition

\footnotetext{
16 Dans une formule qui concerne La Salle de bains, mais possède selon nous une portée autrement générale, Bruno Blanckeman écrit joliment: « Peinture semi-abstraite d'un état d'abstraction partielle au monde » (2002, p. 68).
} 
des désirs, des motivations individuelles ou collectives, des projets ou projections rationnels; c'est une disparition de la part de raison et de la part de désir de l'être, de son histoire, de ses blessures, de son inscription dans un temps et un lieu qui lui disent qui il est. C'est une sorte de gigantesque anesthésie de tout ce qui, d'ordinaire, fait l'individu, le sujet, l'être. Cette expérience non narrative du réel se fait dès lors, par ce biais, première modalité d'un oubli de soi.

Les paramètres descriptifs évoqués ensuite sont la seconde modalité de cet oubli de soi : le réel ne dit plus ni la culture ni l'histoire intime des descripteurs, il en nie la pertinence, sinon l'existence, à même ses formes lumineuses et ses clartés atténuées, à même ces narrations du sensible ${ }^{17}$. Et la disparition de notre reflet dans l'habituel miroir du monde prononce notre propre effacement. Effacement qui se lit encore dans ces narrateurs et personnages anonymes, dans ces lieux romanesques impersonnels (de la chambre d'hôtel au stationnement de supermarché), dans ces êtres, pour la plupart, sans passé ni avenir. Enfin, en dernier retournement, cet effacement se fait aussi élaboration romanesque contre le pouvoir du temps, l'oubli de soi devenant un rempart contre l'inscription mortifère de soi dans le temps, contre la douleur de la lente et inévitable dissolution des êtres et des choses dans l'irréversible cours des jours.

\footnotetext{
17 À propos de ce déplacement de la logique cognitive de la narration vers la rationalité affective, on se reportera avec un profit substantiel, à Frances Fortier et Andrée Mercier ( $«$ La narration du sensible dans le roman contemporain », 2004).
} 


\section{Bibliographie}

ADAM, Jean-Michel et André PETITJEAn, Le Texte descriptif, Paris, Nathan, 1989.

BAlzaC, Honoré de ([1835] 1961), Le Père Goriot, Paris, Librairie Générale Française.

BlANCKEMAN, Bruno (2002), Les Fictions singulières. Études sur le roman français contemporain, Paris, Prétexte éditeur.

BoULAY, Bérenger (2008), « Histoire et narrativité. Autour des chapitres 9 et 23 de La Poétique d'Aristote", http://www.fabula.org/atelier.php?Histoire et narrativit $\% 2$ 6eacute $\% 3 \mathrm{~B} \% 2 \mathrm{E}$ Autour des chapitres $\% 39$ et $\% 32 \% 33 \mathrm{~d}$ e La Po\%26eacute\%3Btique d\%27Aristote (page consultée pour la dernière fois le 28 mai 2008

Huglo, Marie-Pascale (2007), Le Sens du récit. Pour une approche esthétique de la narrativité contemporaine, Villeneuve d'Ascq, Presses Universitaires du Septentrion.

OUELLET, François (1996), «Le Regard de Bataille et la pensée de Valéry sous le ciel étoilé de Minuit », Tangence, $n^{\circ} 52$, septembre, p. 25-39.

RICOEUR, Paul (1984), Temps et récit. Tome II. La configuration dans le récit de fiction, Paris, Seuil.

TouSSAINT, Jean-Philippe (1985), La Salle de bain, Paris, Minuit;

- (1988), L'Appareil photo, Paris, Minuit;

- (1991), La Réticence, Paris, Munuit;

- (1992), Monsieur, Paris, Minuit;

— ([1997] 2002), La Télévision, Paris, Minuit, coll. « Double»;

- (2000), Autoportrait (à l'étranger), Paris, Minuit;

- (2002), Faire l'amour, Paris, Minuit;

- (2005), Fuir, Paris, Minuit.

VIART, Dominique et Bruno VERCIER (2005), La Littérature française au présent. Héritage, modernité, mutations, Paris, Bordas. 


\title{
Résumé
}

Le présent article a pour objectif de mettre au jour certains traits de la poétique narrative et de la poétique descriptive de Jean-Philippe Toussaint ainsi que l'expérience (fictive) du réel qu'ils impliquent. Sur le plan narratif, on essaie de détailler, d'une part, le rapport trouble que les personnages entretiennent avec l'agir comme manière d'imposer un ordre au réel et, d'autre part, la faible configuration des intrigues romanesques. Sur le plan descriptif, on s'emploie à identifier les deux principaux rapports au réel: euphorique lorsque le personnage peut y observer formes, mouvements et lumières intemporels et sans lien avec les destins humains individuels ou collectifs; dysphorique lorsque l'objet de la description devient le lieu d'inscription du passage du temps et la marque visible de l'inévitable déliquescence des êtres et des choses. On tente de montrer enfin que ces pratiques narratives et descriptives imposent une expérience du réel comme oubli de soi précisément parce que, chez Toussaint, cet oubli se fait rempart contre l'action corruptrice et mortifère du temps.

\begin{abstract}
The purpose of this article is to bring to light certain characteristics of the narrative and descriptive poetry of JeanPhilippe Toussaint as well as the poetry's related (fictional) experiences of the real. From a narrative standpoint, we seek to detail, on one hand, the characters'troubled relationship with the notion of being able to impose order on the real through their actions, and on the other hand, the weakness of the plot structures. With respect to the descriptive elements, we seek to identify the two principle relationships to the real; the first consisting of euphoria when the character observes forms,
\end{abstract}


movements and lights that are a-temporal and without link to individual or collective human destiny; the second consisting of dysphoria when the object of the description becomes the place where the passage of time and the inevitable decay of all beings and things are inscribed. Lastly, we attempt to show that these narrative and descriptive practices impose an experience of the real that is based on self-neglect (or the forgetting of self), since, in the work of Toussaint, this neglect is a veritable rampart against the corrupting and mortifying action of time. 